\title{
Syndrome definitions for drug overdose: How far down the rabbit hole do we go?
}

\author{
Alana M. Vivolo-Kantor, Brooke Hoots \\ Division of Unintentional Injury Prevention, Centers for Disease Control and Prevention, Atlanta, Georgia, United States \\ Objective
}

To discuss the process for developing and revising suspected drug overdose queries in syndromic surveillance (SyS) systems.

Introduction

State and local jurisdictions have been exploring the use of SyS data to monitor suspected drug overdose outbreaks in their communities. With the increasing awareness and use of SyS systems, staff from the Centers for Disease Control and Prevention (CDC) worked to develop several queries that jurisdictions could use to better capture suspected drug overdose visits. In 2017 , CDC released their first two queries on heroin overdose and opioid overdose, followed in 2018 by stimulant and all drug overdose queries. Over time, and with the assistance from the SyS community and the CDC-funded Enhanced State Opioid Overdose Surveillance (ESOOS) state health departments, CDC has revised the queries to address suggestions from jurisdictions. However, it's not clear how often and in what way the syndrome definitions are updated over time. This is particularly true as new drugs emerge and the names of those drugs are integrated into syndrome definitions (e.g., recent "Spice" and "K2" synthetic cannabinoid outbreaks).

\section{Description}

This roundtable will provide a forum for national, state, and local users of SyS and drug overdose syndrome queries to discuss the process of query development, with an eye towards determining when a definition is "good enough." CDC staff will facilitate the discussion and present the current portfolio of drug-related overdose queries. Participants will be encouraged to provide feedback on the queries, share what has been/has not been working in their jurisdiction with regard to syndrome query development, and discuss the process for revising queries as the epidemic evolves. The focus of this roundtable will be on suspected drug overdose query development and revision with emergency department SyS data. 